\title{
Surface Profile Based on Sensor Fusion
}

\author{
Cristina Santos, Jaime Fonseca, Paulo Garrido, Carlos Couto \\ Department of Industrial Electronics \\ School of Engineering - University of Minho \\ Braga-Portugal
}

This paper reports a sensor system which it has been designed and constructed to acquire the profile of surfaces. This system is based in a CCD camera for object boundary-determination mounted over a robot manipulator shoulder and ultrasonic sensors for depth measurement mounted on a fixture at the wrist of the manipulator. It has been used an average weighted by degrees of confidence for raw sensor data fusion, based on a heuristic set of rules.

\section{INTRODUCTION}

From an economical point of view may be interesting to replace a single highly accurate but expensive sensor by several less precise low-cost sensors requiring with additional post-processing electronics. Using several lowcost sensors combined with intelligent post-processing can compensate the its low accuracy. These sensors can be either of the same type or give complement information. With the same type of sensors the goal is to increase the quality of the resulting sensor information. Of course, the improvement must be reasonable compared with the increasing complexity of the measurement system in order to keep the overall cost still attractive. As the computing power cost is decreasing everyday and the low cost sensors is bound to proliferate in the near future, multisensor systems and sensor fusion techniques bound to became more and more popular.

Several sensor fusion methods have been reported that deal with this problem. Durrant-Whyte has developed a Bayesian estimation technique for combining touch and stereo sensing[8]. Tang and Lee proposed a generic framework that employs a sensor-independent, feature-based relational model to represent information acquired by various sensors[19]. In[9], a Kalman filter update equation was developed to obtain the correspondence of a line segment to a model, and this correspondence was then used to correct position estimation. In[18], a extended Kalman filter was used to manipulate image and spatial uncertainties.

The work described in this paper is concerned with the development of a robot based system to acquire the profile of a surface. A PUMA 560 manipulator was equipped with a CCD video camera and one ultrasonic sensor in the shoulder and a set of four ultrasonic sensors in the wrist, to acquire data for internally representing the geometry of the part's surface, exploiting the mobility of the robot. The camera defines the work area while the sensors enable to get the surface profile. We used an average weighted by degrees of confidence for raw sensor data fusion to obtain more reliable representation in profile surface acquisition.

This paper is organised as follows: Section 2 describes our hardware configuration, Section 3 describes the software procedures which implement image acquisition and depth map filling of the part to be acquired, Section 4 presents tests and results and, Section 5 presents the conclusions and future work suggestions.

\section{HARDWARE CONFIGURATION}

\subsection{Sensor System Design}

The sensor system consists of a National Electronics monochrome CCD video camera and five ultrasonic sensors. The CCD camera and one ultrasonic sensor are mounted over the shoulder of the Puma, both horizontally aligned, to enabling a circular scanning around the robot stand. The other four ultrasonic sensors are mounted on a fixture at the wrist of the manipulator, hence benefiting from all degrees of freedom of the robot. 
The disposition of these ultrasonic sensors, relative to the robot grip's axis, is a square as presented in the figure 1. The distance between them is few centimetres.

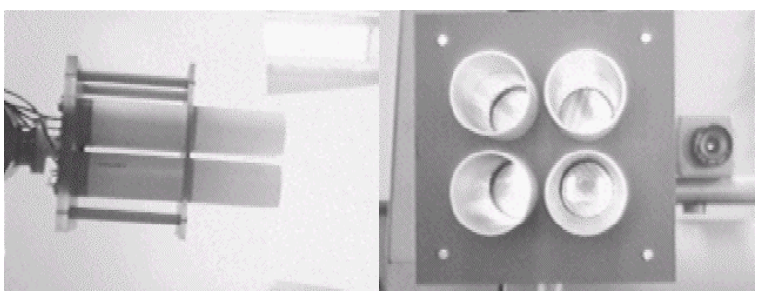

Fig.1 - Location of the sensors. a) Side view. b) Front view

The sensors used in this work are adopted from Polaroid Ultrasonic Ranging Units, which have a range of about $0.35 \mathrm{~m}$ to $4 \mathrm{~m}$. The ultrasonic transducers have emission frequencies of $63 \mathrm{kHz}$ and are controlled by a specific kit provided by Polaroid Corp. This kit is based in the Intel 80C196 microprocessor and is easy to configure by software. It is possible to configure the following parameters: transmit frequency, pulse width, blanking time, amplifier gain, sample rate and trigger source internal/external. This kit is connected with external world by RS-232. To avoid the possible interference from the emission and echo waves, the sensors are triggered sequentially, leaving just one unit emitting at a time.

The CCD camera, with a resolution $500(\mathrm{H}) \times 582(\mathrm{~V})$ is connected to the low-cost Creative's Video Blaster acquisition board inside of the Pentium PC. The software for the image processing was developed using Borland $\mathrm{C}$ under Windows environment.

\subsection{Limitations of Ultrasonic Sensors}

The basic principle of distance measurement using ultrasonic sensor is to record the time between the emission of the transmission wave and the reception of the returned echo. However, due to the wide beam opening angle of the ultrasonic transducers, a measurement error will occur if the emitted wave is not perpendicular to the target object. As depicted in the figure 2., the distance between the sensor and a flat wall should be $\overline{O A}$. But we will detect the distance $\overline{O A^{\prime}}$, because this point provides the first returned echo. The position measured in this case is then given by $\mathrm{A}$.

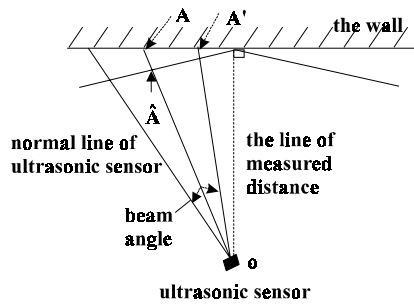

Fig. 2 - The influence of the beam opening angle when scanning a wall with an ultrasonic sensor

Therefore, there is a difference between the location of the perceived wall and the location of the actual wall. This error caused by the beam opening angle affects the accuracy of the environment perception. We found that the maximum beam opening angle for the sensors is about $22^{\circ}$. On the other hand, the optimal angle between the line of emission and the surface of the measurement object is $90^{\circ}$. However, if the incident angle exceeds the maximum value $\alpha$, the echo may be received after multiple reflections. The detected data will then be erroneous. Therefore, ultrasonic sensors can easily measure the distance to an object, but they have poor angular resolution, due to the beam opening angle and to the spurious reflections phenomenon.

The methodology followed to decrease the beam opening angle was to increase the frequency $(63 \mathrm{kHz})$ and to put a tube $20 \mathrm{~cm}$ long with $4 \mathrm{~cm}$ diameter in front of the ultrasonic sensors. 


\subsection{Can Network}

The computing hardware includes two CAN boards, the Universal CAN I/O board outside the computer and the PC-CAN Interface PCIO2 inside the PC. Both boards are based on the Intel 80592, products of STZP (Steinbeis Transferzentrum Prozessautomatisierung).

The Universal CAN I/O board deals with the Polaroid 's kit receiving the data sent and assuring the sequential triggering of the transducers. In reply to a trigger signal several measurements are made and the average value is calculated. This pre-processed data is then sent to the PC via the CAN net at $1 \mathrm{Mb}$ baudrate. This CAN board has the following features: 16 digital inputs, 16 digital output, 8 analogue inputs and 2 pulse with modulated output.

The software was developed in IAR C for the Universal CAN I/O board and in Borland C for the PCIO2 board.

The software for communication is developed in IAR C and Borland C for the Universal CAN I/O board and PCI02 board.

\subsection{PUMA 560}

The PUMA 560 is a six-joint industrial robot manipulator, whose original UNIMATE MARK II controller includes a DEC LSI/11 processor and six digital and analogue servo boards. Interaction with the user is possible through the VAL-II operating system. The LSI/11 interprets VAL-II statements and generates joint interpolated co-ordinates, sending them to the six 6503 microprocessors. The PUMA 560 original control architecture is depicted in figure 3 .

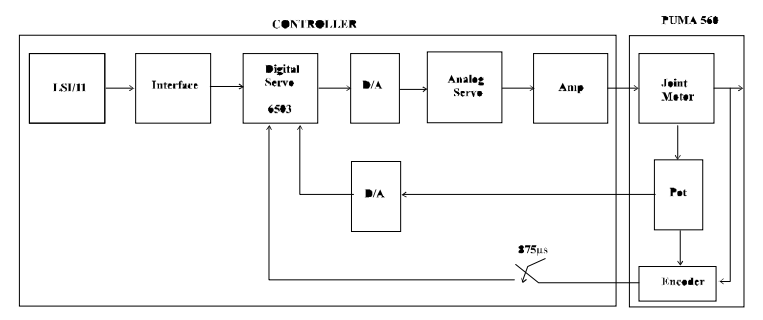

Fig. 3 - Original control architecture

This architecture offers many difficulties when used for high level control tasks, since the operating system VAL-II is based on a closed architecture. Resources to accomplish sensing, such as vision and force, are not supported. Also, the joint controllers have fixed gains and it is impossible to change them or to change the way the controller generates the paths following its internal interpolators. Hence, the implementation of new strategies for each of the joint controllers, the generation of trajectories or task planning algorithms are not possible.

The solution adopted to avoid the above mentioned limitations, was to replace the operating system VAL-II and most of the manipulator control hardware. The new installed hardware gives direct access to the joint positions and bypasses the old joint controllers. This new system is based on the Trident Robotics cards TRC004, TRC100, and TRC041 (Puma cable card set). The system composed by the TRC004 and TRC100 cards plus a personal computer (PC), replaces the old system composed by the LSI/11 processor, the VAL-II operating system and the joint controllers. The remaining components from the original architecture are the incremental encoders, potentiometers, power amplifiers and analogue servos.

The TRC004 is a general purpose interface board for servo applications. It provides eight channels of buffered analogue output, eight channels of analogue input, six quadrature shaft encoder channels, six bits of discrete input, six bits of discrete output and a watchdog timer. Physically, the CPU, RAM, EPROM, serial controller, interface cards, and joint servos are all removed from controller backplane. In their place, the TRC004, a single twelve-inch by nine-inch (approximately) multi-purpose I/O card is mounted and wired to the backplane by TRC041 card.

The TRC100 is a general purpose RISC processor board for servo control and data acquisition applications. With its local I/O bus the processor operates either standalone or in an ISA or EISA backplane. For real-time 
control, the code is either downloaded from a host computer or stored in EPROM. In our case, this card provides an interface between the TRC004 and the ISA bus of the PC.

The block diagram of the new architecture is represented in figure 4.

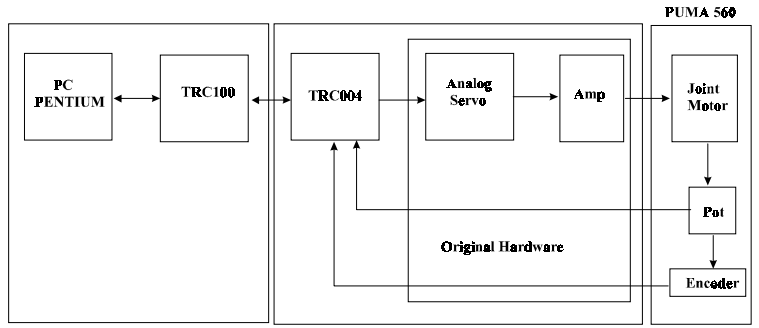

Fig. 4 - New architecture

The interface between the TRC100 and TRC004 is accomplished by a software developed by Trident Robotics (a DLL for Windows environment and some software placed in the EPROM of TRC100).

\section{PROFILE SURFACE}

In this section we will describe all steps needed to acquire surface profile: object search and robot positioning, surface scanning for depth acquisition.

The robot is positioned at the centre of a ring table, in which objects whose surface has to be acquired can be positioned. This table has $100 \mathrm{~cm}$ of height, $95 \mathrm{~cm}$ of internal ratio and $125 \mathrm{~cm}$ of external ratio.

\subsection{Search for the object and robot positioning}

The incremental rotational movement of the robot's base and the processing of the acquired images allow the location of the object performing the search process.

After the object detection, the system stops the rotational movement of the robot and centres the object in the vision plan of the camera, as shown in the figure 5 . Next, the dominant points of the contour are extracted in order to create a $2 \mathrm{D}$ representation of the part's surface.

The extraction of the dominant points is implemented by the use of the combination of two algorithms. The first algorithm performs segmentation, which is achieved by Otsu global thresholding [2], selected on the basis of a comparative study covering Otsu, Maximum Entropy, Uniform Error and Minimum Error Threshold selection methods described in [11]. The second algorithm, developed for the extraction of the dominant points, is again a combination of two algorithms. The first marks pixels as candidates for dominant points and it is an improved version of the classical splitting method presented by Duda and Hart [13]. The second provides the selection and is based on slope[17]. This arrangement was devised to provide a process for dominant points extraction suitable for most different sorts of object shapes. The dominant points are depicted in the figure 5 .

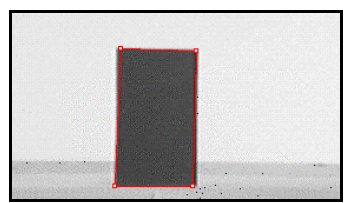

Fig. 5 - Object extraction from background: Dominants points

The method implemented for calibration allow the object to present the correct dimensions since positioned over the worktable. The ultrasonic sensor mounted over the camera measures the distance between the camera and the object's centroid.

The robot is moved to a fixed distance of the table at the table height and in the direction of the centroid's line.

The process described confines the work area of the manipulator, and sets the system ready for horizontal object scanning. 


\subsection{Surface scanning for depth acquisition}

The $3 \mathrm{D}$ acquisition is accomplished by making the manipulator scan the 2D shape with its ultrasonic sensors. The overall result of this task is the building of a surface map that shall support the generation of profile surface. The $y$ co-ordinates that the image acquisition obtained for the part's dominant points are subjected to perspective errors. Those values were based on the depth measurement given by the ultrasonic sensor mounted on the top of the video camera, which isn't the depth of all points but approximately of the object's centroid, as the figure 6.a) illustrates. The only co-ordinate that the system can consider without error is the $z$ co-ordinate as the object is positioned over the table, where $z$ co-ordinate is known and fixed.

This way, when acquiring points in the boundary region an uncertainty margin must be considered and the found boundaries are augmented by a certain default value, as figure 6.b) illustrates.

This contour enlargement minimises the perspective and calibration errors because the true values of $x, y$ coordinates of the boundary points will be validated later by the scanning with ultrasonic sensors.
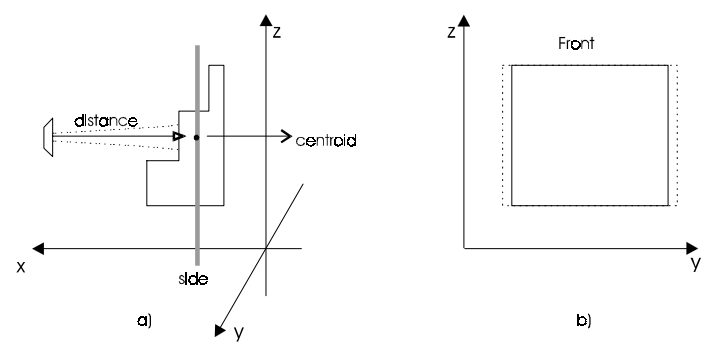

Fig. 6 - Object acquisition a) Side view b) Front view and contour enlargement

The algorithm implemented calculates the next position for acquisition using a fixed step. This step has the same value for the $z$ and $y$ co-ordinates. For each horizontal scan line, the start point is always defined by one extreme of the boundary calculated and the robot will step along evenly spaced points, till the end of the scan line. The definition of this step is done "a priori" and it depends on the desired precision for acquisition and the minimum resolution allowed to the surface. We used a fixed step $s$ given by $s=\frac{d s}{5}$, where $d s$ is the distance between the sensors.

In order to calculate the joint angles needed to position the robot in the next position, we used inverse kinematics since the manipulator's position is known. This feature is given by the new architecture that allows the necessary feedback to control the robot. For the implementation of inverse kinematics we used Borland C, based on the Robotics Toolbox of Peter Corke [14].

Assuming that the surface does not present significant changes in the square defined by the four ultrasonic sensors, it seems reasonable to accept that the average of the sensors measurements can be assigned to that square centre point of the part's surface. This point lies on the grip axis, i.e., in the axis passing through the centre of the sensor arrangement. Following this procedure, to maintain the resolution of the depth map, the speed of acquisition must be traded-off against sensor fusion in order to get greater accuracy. It is always a startstop process, as the system needs the sensor data to decide what to do next.

Besides spatially averaging the ultrasonic readings, weights were introduced affecting each of the sensor measurements. These weights are to be interpreted as degrees of confidence, based on a heuristic set of rules [3] [5]. The sensor fusion problem is treated like if a group of human beings is positioned in front of a decision problem. For each point, after reading the data and the parameters defining the situation of acquisition, the system introduces a degree of confidence to each measure collected, where a high value denotes high confidence that the read value is correct. This degree of confidence takes values between $[0,1]$ and can be interpreted as a probability given to the veracity of the proposition "The measurement read by the sensor $i$ is correct".

The distance assigned to the surface's point lying at the grip axis for the scanning position $n$, was denoted $M$ and is given by: 


$$
M(n)=\frac{\sum_{i=1}^{4} m_{\text {sensor }}(i) \times d c(i)}{\sum_{i=1}^{4} d c(i)},
$$

where $i$ is the identifier of a sensor, $m_{\text {sensor }}(i)$ the measurement given by the sensor $i$ and $d c(i)$ its degree of confidence.

It is worth to note that the $\mathrm{m}_{\text {sensor }}$ values are validated before this calculation according to the range of values physically possible. A set of tests is applied to each measurement to determine if it corresponds to a point inside or outside the object and to testify if the received value is consistent with the physical set-up. Sensors whose measurements are not validated do not enter into the average calculation. The parameter $d c$ is established as the result of the product of 3 partial degrees of confidence

$$
d c(i)=d c_{-} 1(i) \times d c \_2(i) \times d c \_3(i)
$$

which heuristically are defined to tackle different aspects of the problems posed by using multiple ultrasonic sensors to measure distance. Each of them is established according to the following heuristic.

- A read value that presents a different value comparatively to the calculated by the system as expected should have a lower weight in the average. This degree of confidence $1, d c_{-} l$, shows the alignment of the sensor values with those predicted by a least-squares linear predictor/ estimator of the surface's inclination.

The calculus of the surface slope is used to predict the surface value, using the past story. The three last values of the average, $M$, are stored in a temporal window. If there is a big variation between the average value calculated using the measurements and the expected value, then the temporal window and the expected value should be reinitialised, because there has been a change in the surface characteristics.

- The read value should have less weight in the average calculation if, when compared to other sensors under the same circumstances, it presents a deviation greater then the one allowed. This sensor should have its rate of errors incremented. This deviation and the rate of errors determine the degree of confidence $2, d c \_2$. This $d c_{\_} 2$ expresses the agreement of each sensor measure with the measures of the other 3 . The underlying assumption is that one is dealing with surfaces of smooth and small curvature.

- The fact that a sensor is positioned near the boundary region introduces the degree of confidence $3, d c_{-} 3$. This was specifically set-up to account for the detection of the part's edges. Its calculation uses a sigmoid function to approximate the probability distribution that the grip is pointing effectively to the part.

\section{TESTS AND RESULTS}

We used Matlab 4.2c to visualise the obtained surface. The algorithm we used to visualise the surface uses splines to construct the surface. Although this could be an advantage, for this specific case it isn't because the interpolation function opposes itself to big variations, trying to minimise the second derivative. Because of this the acquired surface presents a rounded aspect.

The experimental results of this process were obtained through the acquisition of a rectangular flat part with $20 \times 20 \mathrm{~cm}$, where the disposition of the surface relatively to the acquisition plan varied from $0^{\circ}$ to $20^{\circ}$, as displayed in the figure. In a second experience we put another piece, $9 \mathrm{~cm}$ thick with $10 \mathrm{~cm}$ of height and $20 \mathrm{~cm}$ of width in front of the latter one, as showed in the figure 7.b). In this way, we obtained a piece with a relief in the order of $9 \mathrm{~cm}$. 

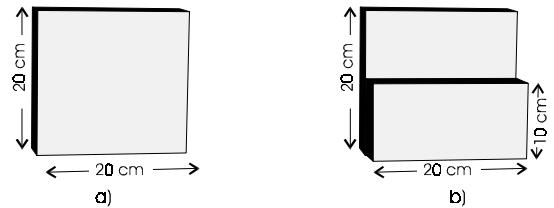

Fig. 7 - Pieces for acquisition a) Flat piece b) Piece with some relief

The figure 8 shows the surface obtained using an average weighted by degrees of confidence. The acquired surface was a flat surface with $20 \times 20 \mathrm{~cm}$, with an angle of $0^{\circ}$ relatively to the plan of acquisition and $43 \mathrm{~cm}$ away from the ultrasonic sensors.

An analysis of the figure 8 shows that the shape of the object surface and its description were well retrieved.

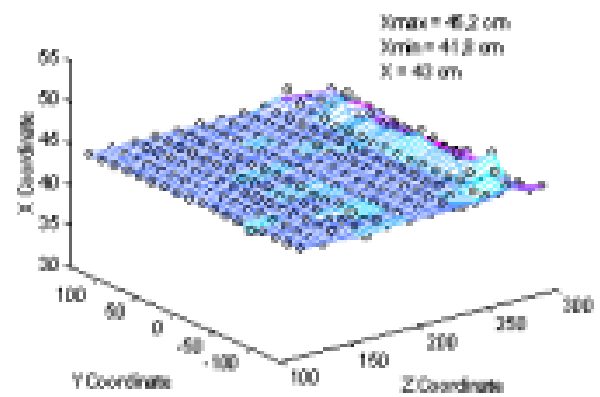

Fig. 8 - Experimental results for the map of a rectangular flat part with $0^{\circ}$ of inclination

In the figure 9 we can see the obtained surface when the flat surface was positioned with $20^{\circ}$ relatively to the scanning plan. As the maximum angle of incidence for these sensors is $25^{\circ}$ the obtained results aren't unexpected.

The acquisition of a surface with $9 \mathrm{~cm}$ of relief shows that the fusion system minimises the slope of the surface, opposing to big slopes.

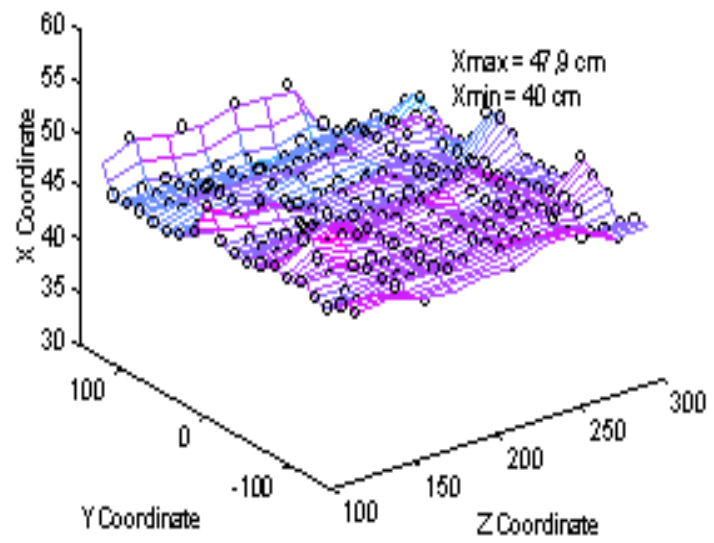

Fig. 9 - Acquisition of the flat surface with $20^{\circ}$ of inclination relatively to the plan of acquisition 


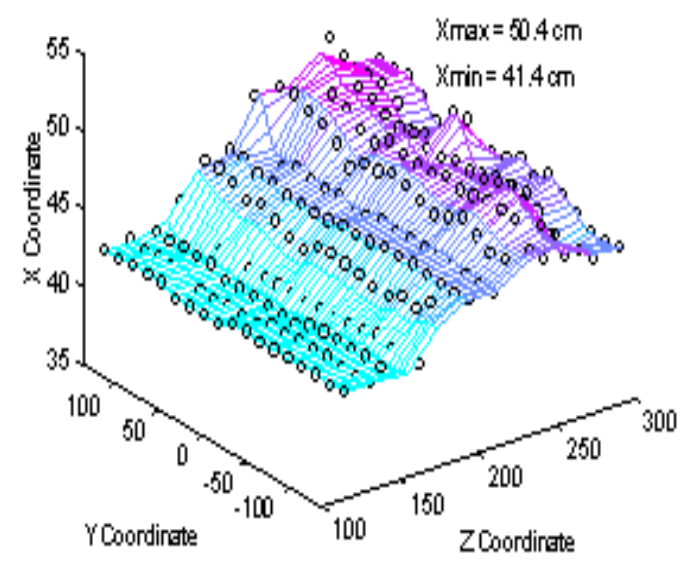

Fig. 10 - Acquisition of a surface with $9 \mathrm{~cm}$ of relief

\section{CONCLUSIONS AND FUTURE WORK}

A sensor system has been designed and constructed to acquire the profile of surfaces, based in a CCD camera for object boundary-determination and ultrasonic sensors for depth measurement. In order to reduce the measurement error resulting from the beam opening angle we covered the ultrasonic sensors with a tube of 20 $\mathrm{cm}$ and incremented the working frequency.

We used an average weighted by degrees of confidence for raw sensor data fusion, based on a heuristic set of rules. Practical experiments showed that the measurement errors when conventional ultrasonic sensors are used alone can be reduced using this multisensor system.

The experimental tests made with the new architecture for the PUMA 560 manipulator showed that it is possible to implement control actions more sophisticated comparatively to the old architecture.

The CAN network showed that it is highly reliable even when used under noisy environments. This feature is spreading CAN network in industrial environments.

This study demonstrates an encouraging approach to integrate several sensors at different levels. Surfaces more complexes, such as big inclinations relative to the plan of acquisition or big variations of depth inside the object, have to be treated in other ways. In order to reduce the angle of inclination relative to the acquisition plan, the grip axis should be normal to the estimated surface curvature. In other situations other kind of more directional sensors, like infrared sensors, should be used and their data included in the sensor fusion.

There is a lot of future work to be done in this project, including improvements to the results already obtained. Current work is in progress for the adaptation of the depth measuring grid resolution to the surface curvature.

To minimise the movement duration of the manipulator, direct control of the joints is being considered, eventually with non-linear techniques. These sophisticated control actions are now possible because the new architecture provides all information necessary for these tasks. Regarding the geometry's acquisition time, high level techniques as clustering shapes and the correspondent class creation, as well as learning on geometry is being envisaged. 


\section{REFERENCES}

1. A. Watt, 3D Computer Graphics, Addison Wesley, 1993.

2. C. Santos, N. Monteiro, J. Fonseca, P. Garrido and C. Couto, "Control of a Robot Painting System Using the Multiresolution Architectural Principle", in Proceedings of the IEEE International Symposium on Industrial Electronics - ISIE97, Guimarães, Portugal, 7-11 July, 1997, pp. 672-677.

3. C. Santos, Uma exploração do Modelo de Referência RCS e da Fusão Sensorial em Robótica, Master Thesis, Guimarães, Universidade do Minho, October 1997

4. CAN - Komponentem System Tools, Steinbeis Transferzentrum Prozessautomatisierung , 1996

5. E. Piat and D. Meizel, " Range Data Fusion for occupancy grid building," in Proceedings of the $4^{\text {th }}$ International Symposium on Intelligent Robotic Systems '96, pp. 353-360, Lisbon 1996.

6. Foley and Hughes, 3D Computer Graphics \& Principle, Addison Wesley, 1992.

7. G. Blais and M.D. Levine, "Registering Multiview Range Data to Create 3D Computer Objects". IEEE Transactions on Pattern Analysis and Machine Intelligence, 17 (8) p.820-824.

8. H. F. Durrant-Whyte, "Consistent integration and propagation of disparate sensor observations". Int. J. Robot., Res., vol. 6, no. 3, pp. 3-24, 1987.

9. J. L. Crowly, "World modeling and position estimation for a mobile robot using ultrasonic ranging". in Proc. IEEE Int. Conf. Robot., Automat., 1989, pp. 674-680.

10. Kai-Tai Song and Wen-Hui Tang, "Environment Perception for a Mobile Robot Using Double Ultrasonic Sensors and a CCD Camera". IEEE Transactions on Industrial Electronics, Vol. 43 NO.3 June 1996, p.372-379

11. N. Monteiro, Processamento e Análise de Imagem para para a Apreensão e Descrição de Contornos dos Objectos, Relatório de Estágio, Universidade do Minho, Departamento de Electrónica Industrial, Maio 1997

12. Nuno Moreira, Paulo Alvito e Pedro Lima, "First Steps Towards an Open Control Architecture for a PUMA 560". in Proc. of the $2^{\text {nd }}$ Portuguese Conference on Automatic Control Porto, Portugal, September 11-13, 1996

13. N. Otsu, "A threshold selection method from grey level histogram". IEEE Transactions on Pattern Analysis and Machine Intelligence, vol. SMC-8, 1978.

14. Peter I. Corke, Robotics Toolbox for use with MATLAB (Release 4), August 1996

15. Polaroid, Ultrassonic Ranging Developer's Kit, Polaroid Corporation, 9/95, USA

16. R. Gonzalez and R. Woods, Digital Image Processing. Addison Wesley, 1992.

17. R. Lima and A. Campilho, "Thresholding selection methods: a comparative study". Proceedings of the RECPAD'94 - The $6^{\text {th }}$ Annual Conference of the Portuguese Association for Pattern Recognition, 1994.

18. T. Skordas, P. Puget, R. Zigmann, and N. Ayache, "Building 3-D edge-lines tracked in an image sequence," in Proc. Intell. Autonomous Systems-2, Amsterdan, 1989, pp. 907-917

19. Y. C. Tang and C. S. G. Lee, “A geometric feature relation graph formulation for consistent sensor fusion,”in Proc. IEEE 1990 Int. Conf. Syst., Man, Cybern., Los Angeles, CA, 1990, pp.188-193. 